\title{
Health-Related Quality of Life Among Older Adults After an Earthquake
}

\author{
Tomoyuki Kawada, MD (1]
}

Key Words: earthquake, health-related quality of life, older adults, risk assessment

I have read with great interest the paper by Wagle et al. dealing with factors affecting health-related quality of life (QOL) among 362 older adults after the earthquake in Nepal. ${ }^{1}$ Questionnaires were applied to measure posttraumatic stress disorder (PTSD) and depression, functional ability, and social support. A multiple linear regression analysis was adopted for the analysis, and standardized regression coefficients of functional status, PTSD, chronic disease, social support, injury, and age for predicting QOL were 0.295, $0.225,-0.168,0.120,-0.104$, and -0.116 , respectively. I have 2 concerns about the study.

First, Hashmi et al. reported the prevalence of psychological morbidity among survivors of the northern Pakistan earthquake. ${ }^{2}$ About half of the survivors had symptoms of PTSD, and more than three-fourths exhibited symptoms of an anxiety disorder at 6 months after the incident. This report presents the significance of health status monitoring for survivors, and a longterm follow-up of survivors might be needed to verify the change of health effects by an earthquake.

Second, Nunnerley et al. conducted a review to evaluate QOL and participation outcomes of individuals with earthquake-related physical injury. ${ }^{3}$ Injured earthquake survivors in developing countries experienced diminished participation and reduced QOL, and functional consequences by the injury and the environmental impact from the earthquake should be simultaneously included for the assessment of QOL after an earthquake.
Finally, keeping QOL of older adults after an earthquake should be considered. Recovery of QOL after an earthquake in older adults might be more difficult than that of younger adults, and maintaining the level of QOL, including physical activity, should be supported to avoid deterioration of health status. ${ }^{4}$

\section{About the Author}

Department of Hygiene and Public Health, Nippon Medical School, Tokyo, Japan.

Correspondence and reprint requests to Tomoyuki Kawada, Hygiene and Public Health, Nippon Medical School, 1-1-5 Sendagi, Bunkyo-Ku, Tokyo, Japan (e-mail: kawada@nms.ac.jp).

\section{Conflict of Interest Statement}

The author has no conflict of interest to declare.

\section{REFERENCES}

1. Wagle S, Amnatsatsue K, Adhikari B, et al. Health-related quality of life after the 2015 Gorkha earthquakes, among older adults living in Lalitpur district of central Nepal. Disaster Med Public Health Prep. 2020. doi: 10.1017/dmp.2019.154.

2. Hashmi S, Petraro P, Rizzo T, et al. Symptoms of anxiety, depression, and posttraumatic stress among survivors of the 2005 Pakistani earthquake. Disaster Med Public Health Prep. 2011;5(4):293-299.

3. Nunnerley J, Dunn J, McPherson K, et al. Participation and quality of life outcomes among individuals with earthquake-related physical disability: a systematic review. J Rehabil Med. 2015;47(5):385-393.

4. Moriyama N, Urabe Y, Onoda S, et al. Effect of residence in temporary housing after the Great East Japan Earthquake on the physical activity and quality of life of older survivors. Disaster Med Public Health Prep. 2017;11(6):701-710. 